\title{
Microfluidic Motion for a Direct Investigation of the Structural Dynamics of Glass-Forming Liquids
}

\author{
Ilenia Viola,* Dario Pisignano, Roberto Cingolani, and Giuseppe Gigli \\ NNL, National Nanotechnology Laboratory of the Istituto Nazionale di Fisica della Materia (INFM), c/o Dip. Ingegneria \\ dell'Innovazione, Universitá di Lecce, via Arnesano, I-73100 Lecce, Italy
}

Glass-forming liquids, polymer solutions, and biofluids have additional inertial and elastic macroscopic degrees of freedom that are related to the elasticity of the molecular coils and affect the determination of the structural dynamical parameters. In this work, we propose a new approach for the direct evaluation of the fundamental material parameters (viscosity, fragility, glass transition temperature) of a viscoelastic liquid in a capillary flow inside a microfluidic device. The proposed technique substantially reduces the complexity of the theoretical analysis and provides an evaluation of the most relevant functional parameters of the fluid dynamics. Moreover, the approach allows the investigation of localization phenomena in geometrical confined systems, such as those required in miniaturized devices.

In recent years, the study of the structural and dynamical properties of disordered systems, such as glass-forming liquids, has attracted increasing interest by virtue of their potential use in functional devices for medical and electronic applications. The capability of manipulating viscoelastic liquids within networks of microchannels is in fact crucial for the fabrication of microfluidic devices, functional components for local flow control ${ }^{1,2}$ such as mixers, diluters, and valves, or lab-on-chip devices such as bioassays and chemical sensors. ${ }^{3,4}$

However, even though a large number of studies have been carried out, a complete understanding of the polymer structural dynamics is still far from being achieved due to the complexity of these systems. As opposed to Newtonian fluids, polymers have in fact additional macroscopic inertial and elastic degrees of freedom, related to the elasticity of the polymer chains, which influences and modifies the small-scale flow., 5 This makes the flow dynamics of molecules nonlinear and the relaxation time parameters dependent on the elongation of the chain. ${ }^{7,8}$ These

* To whom correspondence should be addressed. Fax: +39-0832-298238. E-mail: ilenia.viola@unile.it. URL: http://www.nnl.it.

(1) Beebe, D. J.; Bauer, J. M.; Yu, Q.; Liu, R. H.; Devadoss, C.; Jo, B.-H. Nature 2000, 404, 588-590.

(2) Stroock, A. D.; Dertinger, S. K. W.; Ajdari, A.; Mezic, I.; Stone, H. A.; Whitesides, G. M. Science 2002, 295, 647-651.

(3) Sinton, D.; Ren, L.; Li, D. J. Colloid Interface Sci. 2003, 260, 431-439.

(4) Kenis, P. J. A.; Ismagilov, R. F.; Whitesides, G. M. Science 1999, 285, 8385

(5) Popelar, C. F.; Liechti, K. M. Mech. Time-Depend. Mater. 2003, 7, 89-141. (6) Park, S. J.; Liechti, K. M. Mech. Time-Depend. Mater. 2003, 7, 143-164.

(7) Strobl, G. The Physics of Polymers; Springer: Berlin, 1997.

(8) Groisman, A.; Steinberg, V. Nature 2001, 410, 905-908. properties result in a complex determination of the material parameters identifying the fluid, i.e., shear rate, stress, and characteristic time. ${ }^{9,10}$

Most of the conventional techniques for the analysis of the structural dynamics, such as incoherent quasi elastic neutron scattering, ${ }^{11,12}$ dynamic light scattering, ${ }^{13}$ Raman scattering, ${ }^{14,15}$ dynamical mechanical thermal analysis, ${ }^{16}$ ultrasonic techniques, ${ }^{17}$ and nuclear magnetic resonance, ${ }^{18}$ refer to theoretical models related to the relaxation modes and dynamical properties of the polymer chain at different length or time scales (i.e., EinsteinStokes or Debye relations, Van-Hove function).

For complex systems with a high number of degrees of freedom, these models usually require the solution of a manybody problem. For these reasons, to date, most of the studies on glass-forming liquids have been limited to dilute or semidilute solutions where the number of relevant parameters is reduced and the analysis is strongly simplified. ${ }^{13,18}$

To avoid a many-body approach without extremely straining the experimental conditions, direct studies of the dynamics could be performed by rheology experiments, i.e., viscosity, strain, or normal stress measurements, ${ }^{19}$ or by differential scanning calorimetry for the determination of glass transition temperature or activation energy. ${ }^{20,21}$

However, both these conventional analysis techniques refer to macroscopic systems, neglecting the effects on the structural dynamics induced by the confined environments, which are expected to occur in miniaturized devices, characterized by a high

(9) Balkovsky, E.; Fouxon, A.; Lebedev, V. Phys. Rev. E 2001, 64, 056301.

(10) Amarouchene, Y.; Bonn, D.; Meunier, J.; Kellay, H. Phys. Rev. Lett. 2001, $86,3558-3561$.

(11) Crupi, V.; Magazù, S.; Majolino, D.; Migliardo, P.; Wanderlingh, U. Physica B 1997, 234-236, 256-257.

(12) Teixeira, J.; Zanotti, J. M.; Bellissent-Funel, M. C.; Chen, S. H. Physica B 1997, 234-236, 370-374.

(13) Faraone, A.; Magazù, S.; Maisano, G.; Ponterio, R.; Villari, V. Macromolecules 1999, 32, 1128-1133.

(14) Crupi, V.; Majolino, D.; Migliardo, P.; Venuti, V.J. Mol. Struct. 1998, 448, $261-268$.

(15) Scheyer, Y.; Levelut, C.; Pelous, J.; Durand, D. Phys. Rev. B 1998, 57, $11212-11222$.

(16) Tabellout, M.; Baillif, P. Y.; Randrianantoandro, H.; Litzinger, F.; Emery, J. R.; Nicolai, T.; Durand, D. Phys. Rev. B 1995, 51, 12295-12302.

(17) Baillif, P.-Y.; Tabellout, M.; Emery, J. R. Macromolecules 1999, 32, 34323437.

(18) Magazù, S. J. Mol. Struct. 2000, 523, 47-59.

(19) Schweizer, T. Rheol. Acta 2002, 41, 337-344.

(20) Ping, Z. H.; Nguyen, Q. T.; Chen, S. M.; Zhou, J. Q.; Ding, Y. D. Polymer 2001, 42, 8461-8467.

(21) Naylor, S.; Terrill, N. J.; Yu, Ga-Er; Tanodekaew, S.; Bras, W.; King, S. M.; Booth, C.; Ryan, A. J. Polym. Int. 1997, 44, 371-379. 
surface-to-volume (S/V) ratio. ${ }^{22,23}$ Although in elastic polymer solutions the scale of the characteristic time does not depend on the size of the system, the material parameters and the inertial and elastic turbulence can conversely show strong localization phenomena. ${ }^{24}$ Recent works of Sinton et al. demonstrated, for instance, that the flow loading and dispensing is highly correlated with the hydraulic diameter of the channels. ${ }^{3}$

In this paper, we propose a new approach for a direct evaluation of the fundamental material parameters of viscoelastic liquids, such as viscosity, fragility, and glass transition temperature. ${ }^{25}$ The method uses a theoretical procedure in which the analytical expressions of the fluid displacement in capillaries are simplified by considering the ratio of the capillary dynamics $z(t)$ at two different temperatures. The combination of this theoretical procedure with the direct measurements, by micromolding in capillaries (MIMIC), of the dynamic parameters of polymer fluids filling microchannels, allows us to extract the material functions $T_{0}$ and $D$, the freezing temperature and fragility, respectively. The obtained results show differences, with respect to the values obtained by the rheometric measurements, and underline the dependence of the structural characterization on the experimental setup.

The proposed approach allows us to carry out the analysis of the structural and dynamical properties in confined systems ${ }^{41}$ during operation. Thus, it is especially useful for miniaturized systems, characterized both by a high $\mathrm{S} / \mathrm{V}$ ratio and usually by a fast diffusion regime, where the surface interactions and localization phenomena dominate the capillary dynamics. ${ }^{42}$

All in all, the approach is suitable for systems where the realtime constancy of the work conditions influences the outcome, e.g., in the delivery and in the dynamic mechanisms of a DNA sequencing factory-on-chip. ${ }^{26}$ The model can also be applied to

(22) Zhao, X. M.; Xia, Y.; Whitesides, G. M. J. Mater. Chem. 1997, 7, 10691074.

(23) Ng, J. M. K.; Gitlin, I.; Stroock, A. D.; Whitesides, G. M. Electrophoresis 2002, 23, 3461-3473.

(24) Groisman, A.; Steinberg, V. Nature 2001, 405, 53-55.

(25) Bird, R. B.; Armstrong, R. C.; Hassager, O. Dynamics of Polymeric Liquids; Wiley-Interscience: New York, 1987.

(26) Paegel, B. M.; Blazer, R. G.; Mathies, R. A. Curr. Opin. Biotechnol. 2003 , $14,42-50$.

(27) Bazilevsky, A. V.; Kornev, K. G.; Rozhkov, A. N.; Neimark, A. V. J. Colloid Interface Sci. 2003, 262, 16-24.

(28) Kornev, K. G.; Neimark, A. V. J. Colloid Interface Sci. 2003, 262, 253-262.

(29) Pisignano, D.; Gigli, G.; Visconti, P.; Zocco, A.; Perrone, A.; Cingolani, R. J. Vac. Sci. Technol., B 2002, 20, 2248-2251.

(30) Myers, D. Surfaces, Interfaces and Colloids: Principles and Applications; WileyInterscience: New York, 1999

(31) Delamarche, E.; Bernard, A.; Schmid, H.; Bietsch, A.; Michel, B.; Biebuyck, H. J. Am. Chem. Soc. 1998, 120, 500-508.

(32) Zhao, B.; Moore, J. S.; Beebe, D. J. Science 2001, 291, 1023-1026.

(33) Cook, R. L.; King, H. E.; Herbst, C. A.; Herschbach, D. R. J. Chem. Phys. 1994, 100, 5178-5189.

(34) Mark, J. E. Physical Properties of Polymers Handbook; American Institute of Physics: New York, 1996.

(35) Schug, K. U.; King, H. E. Jr.; Böhmer, R. J. Chem. Phys. 1998, 109, $1472-$ 1477.

(36) Cheng, Z. Y.; Gross, S.; Su, J.; Zhang, Q. M. J. Polym. Sci. B: Polym. Phys. 1999, 37, 983-990.

(37) Graham, M. D. Phys. Fluids 2003, 15, 1702-1710.

(38) Stroock, A. D.; Ismagilov, R. F.; Stone, H. A.; Whitesides, G. M. Langmuir 2003, 19, 4358-4362.

(39) Groisman, A.; Enzelberger, M.; Quake, S. R. Science 2003, 300, 955-958.

(40) Pisignano, D.; Sariconi, E.; Mazzeo, M.; Gigli, G.; Cingolani, R. Adv. Mater. 2002, 14, 1565-1567.

(41) Throughout the manuscript, by confined system we mean a system in which one dimension is considerably smaller than others.

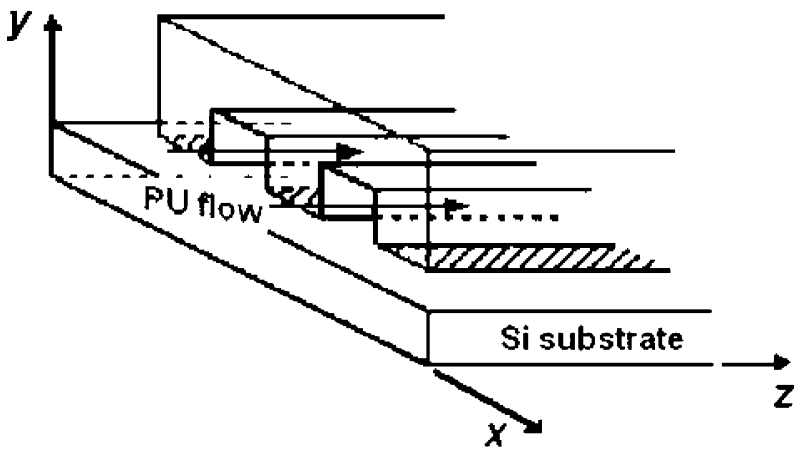

Figure 1. Schematic of the MIMIC process (features not in scale). The conformal contact between the Si support and a PDMS mold induces the PU to flow inside the microchannels.

blood and saliva, which can be considered as mixtures of biopolymers and emulsions..$^{27,28}$

\section{EXPERIMENTAL SECTION}

As a prototype system for our analysis the low-viscosity and high-fragility polyurethane (PU) structural adhesive, NOA72 from Norland, has been used for filling microchannels fabricated by soft lithography techniques (see Figure 1).

Microfluidic Experiments. These were carried out by using poly(dimethylsiloxane) (PDMS) microchannels as horizontal network of $\sim 15-\mu \mathrm{m}$-wide parallel pipes with a period of $30 \mu \mathrm{m}$ and a depth of $1 \mu \mathrm{m}$. The microchannels were defined by placing the PDMS mold in conformal contact with a silicon substrate (Figure 1). A 50- $\mu \mathrm{m}$ resolution ruler has been integrated in the structure to follow, step by step, the filling action through the transparent PDMS element. Details about master fabrication, realization of the elastomeric replica, and the MIMIC procedure are reported in refs 22 and 40 . The driving flow has been followed in real time by an optical microscope. The temperature of the overall system has been varied in the range $292<T(\mathrm{~K})<353$ using a hot plate placed in the bottom side of the $\mathrm{Si}$ substrate (Figure 2).

Rheometric Measurements. These have been carried on NOA72 by a cone-and-plate viscometer from Rheometric Scientific. The plate, with a radius of $R=12.5 \mathrm{~mm}$, is connected to the transducer by a general stem and the angle between the cone and plate is $\alpha=0.02 \mathrm{rad}$. Step shear rate $(\dot{\gamma})$ tests, shown in Figure 3 , were performed over a range $1<\dot{\gamma}<10^{3} \mathrm{~s}^{-1}$. Moreover, by an internal control tool, the sample temperature was changed in a range comparable with the MIMIC data, $295<T(\mathrm{~K})<383$.

\section{THEORETICAL MODEL}

In a general case, for a horizontal capillary system without any significant vertical component, a zero-gravity environment can be assumed (i.e., in our microdevice the vertical dimension $y \ll x, z$; see Figure 1) and the liquid flow is a thermodynamically driven process.

(42) The time condition for a fast diffusion regime is $T_{\mathrm{S}} \gg T_{\mathrm{D}}$, where $T_{\mathrm{S}}$, the surface time, is the average time of the interaction of each molecule with the surface. It is defined as $T_{\mathrm{S}} \simeq \rho^{-1}(V / S)$ with $\rho$ a parameter related to the molecular relaxation rate at the surface. $T_{\mathrm{D}}$ is the molecular diffusion time. According to the Einstein equation, it is $T_{\mathrm{D}} \simeq D^{-1}(V / S)^{2}$. Assuming a surface relaxivity $\rho \simeq 1 \mathrm{\mu m} / \mathrm{s}$, an average diffusion $D=K T / 6 \pi \eta a<10^{-5} \mathrm{~cm}^{2} / \mathrm{s}$, and a molecular diameter $a \simeq 5 \AA$, an upper limit can be evaluated for the fluid viscosity $\eta$ for which the fast diffusion regime takes place. For 100 $\mu$ m-width microchannels, the upper limit is $\eta<500$ counts/s. 

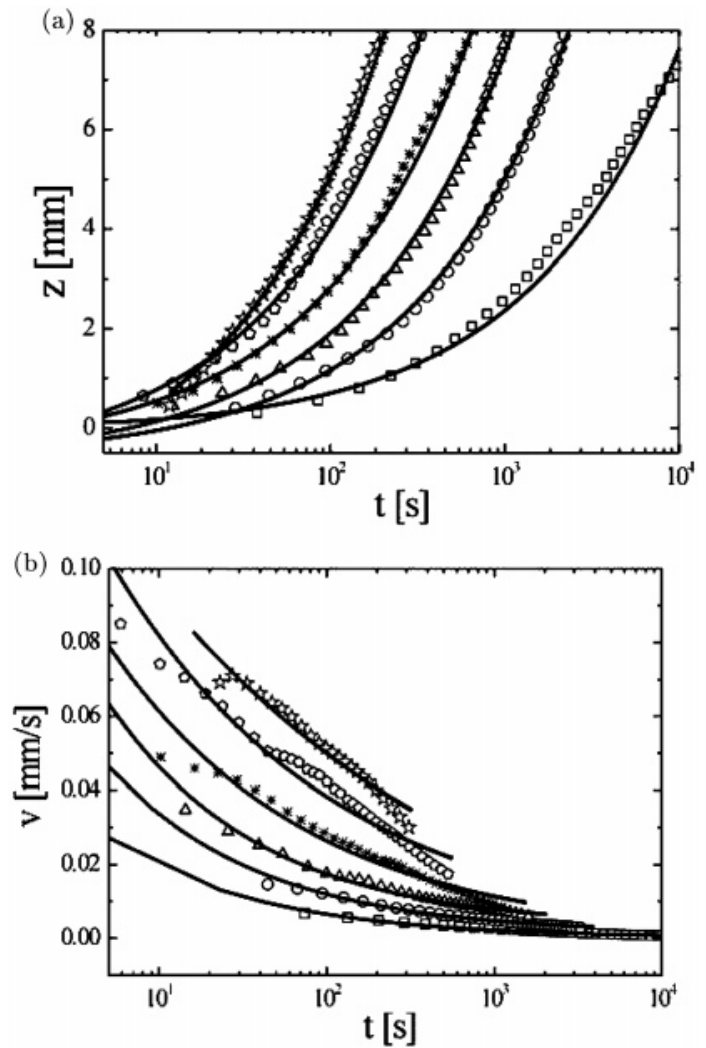

Figure 2. (a) Length coordinate $z(t)$ as a function of time during the driving process of a viscoelastic fluid (PU) inside a PDMS network of micrometer scale capillaries. The experimental data is represented at different temperatures $(T=292(\square), 303(\bigcirc), 313(\triangle), 323(*)$, $343(\diamond), 353(\star) \mathrm{K})$ and the fitting lines $y=y_{0 i}+A_{i} x^{b}$, with $b=0.5$. (b) Flow rate versus time at different temperatures, reported with the same symbols. It can be seen that the velocity of the viscoelastic process also fulfills the -0.5 power law.

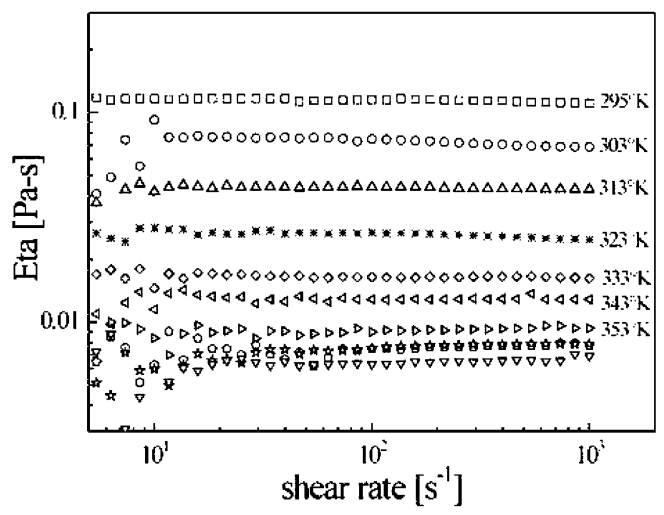

Figure 3. Rheological data of the PU non-Newtonian viscosity $\eta$ at different temperatures, $295<T(\mathrm{~K})<383$. The three bottom curves are respectively obtained at $T=363(\diamond), 373(*)$, and $383(\triangle) \mathrm{K}$. The data have been taken over a shear rate range $1<\dot{\gamma}\left(\mathrm{s}^{-1}\right)<$ $10^{3}$.

Therefore, the capillary motion is primarily influenced by the interfacial mechanical tension and by the curvature in the different phases: liquid-vapor (LV), solid-vapor (SV) or solid-liquid (SL). The difference between the SV and SL interfacial energies affects the contact angle $\theta$ and defines the thermodinamic condition that favors the capillary filling process. ${ }^{29}$ For a simple capillary system this is expressed by the Laplace equation for the pressure drop

$$
\Delta p=\left(2 \gamma_{\mathrm{LV}} \cos \theta\right) / r
$$

where $\gamma_{\mathrm{LV}}$ is the free energy at the liquid-vapor interface and $\theta$ is the contact angle between the liquid and the capillary surface ${ }^{30,31}$ The geometric factor $r$ is the capillary radius for a circular shape section or in the most general case the hydraulic radius, defined by the V/S ratio of the capillary.

In the laminar regime, the equation that controls the motion is

$$
\mathrm{d} z / \mathrm{d} t=G \Delta p / \eta z
$$

where $z$ is the channel length, $\eta$ is the fluid viscosity, $\Delta p$ is the capillary pressure from eq 1 , and $G$ is a geometric factor related to the hydraulic radius. ${ }^{43}$ For a channel of rectangular shape, this can be approximated by

$$
G=\frac{1}{8}\left(\frac{a b}{a+b}\right)^{2}
$$

where $a$ and $b$ indicate the width and the height of a channel, respectively. ${ }^{31}$

The integration of eq 2 defines the displacement of the fluid filling the channel after a time $t$. Thus, the capillary dynamics for the length coordinate is described by a power law model

$$
z(t)=(2 \Delta p G / \eta)^{1 / 2} t^{1 / 2}=A_{z} t^{1 / 2}
$$

which is in agreement with the experimental MIMIC data as could be seen in Figure 2a.

The coefficients $A_{z}$ can be evaluated by a nonlinear regression fit with the function ${ }^{44} y=y_{0 \mathrm{i}}+A_{\mathrm{i}} \mathrm{x}^{\mathrm{b}}$.

The effects of temperature $T(\mathrm{~K})$ are related, in eq 4 , to the $T$-dependence of the inherent time scale and thus of the viscosity $\eta$, considered as the macroscopic manifestation of the stretching of the polymer molecules, ${ }^{5,10}$ as shown in Figure 3, where rheological data of non-Newtonian viscosity at different temperatures are reported. Such a $T$-dependence can be described by the isobaric Vogel-Fulcher-Tamman (VFT) model ${ }^{33}$

$$
\eta(T)=\eta_{0} \exp \left(\frac{D T_{0}}{T-T_{0}}\right)
$$

According to the free volume model, $T_{0}$ in eq 5 is defined as the freezing temperature (also known as Vogel temperature) at which the free volume approaches zero and hence the viscosity diverges toward very high values, ${ }^{45} \eta_{0}$ is proportional to the viscosity in

(43) Noticeably in the dynamic eq 2 the term $G / \eta z$ is responsible for the Poiseullian friction, whereas $\Delta p$ accounts for the capillary driving and the wetting forces. ${ }^{27}$ Moreover, the effects of viscous friction on the driving force are secondary compared with the wetting, inertial and elastic ones. At a submillimeter scale, the liquid behavior is, in fact, significantly affected by surface properties, by advancing contact angle, particularly on the hydrophobic region, and by channel depth. ${ }^{32}$

(44) The term $y_{0 i}$ of the fitting function is introduced in order to account for the measurement beginning.

(45) The $T_{0}$ value is usually related to the glass transition temperature $T_{\mathrm{g}}$. In particular, it comes out $T_{0}=T_{\mathrm{g}}-(30 \div 70) \mathrm{K}$, where $T_{\mathrm{g}}$ indicates the temperature at which, conventionally, $\eta\left(T_{\mathrm{g}}\right)=10^{13} \mathrm{cP} .^{34}$ 
Table 1. Coefficient Values $A_{i}$ of the Nonlinear Fitting Equation $y=y_{0 i}+A_{i} x^{b}$, Where $y_{0 i}$ Is the Offset Terma

\begin{tabular}{|c|c|c|c|}
\hline $\begin{array}{c}y_{0 z} \\
(\mathrm{~mm})\end{array}$ & $\begin{array}{c}A_{z} \\
\left(\mathrm{mms}^{-1 / 2}\right)^{b}\end{array}$ & $\begin{array}{c}y_{0 \mathrm{v}} \\
\left(\mathrm{mms}^{-1}\right)\end{array}$ & $\underset{\left(\mathrm{mms}^{-1 / 2}\right)^{b}}{A_{\mathrm{v}}}$ \\
\hline 2 & $0.080 \pm 0.0$ & $(10 \pm 2) \times 10^{-5}$ & 0.06 \\
\hline 0.01 & $0.200=$ & $10^{-5}$ & 0.001 \\
\hline $70 \pm 0.02$ & $0.260 \pm 0.001$ & $(300 \pm 8) \times 10^{-5}$ & 0.001 \\
\hline+00 & $0.330 \pm 0.002$ & $(70 \pm 4) \times 10^{-4}$ & 0004 \\
\hline 2 & $0.500 \pm 0.003$ & 6) $\times 10^{-4}$ & 0.2 \\
\hline$-1.60 \pm 0.03$ & $0.700 \pm 0.003$ & $(20 \pm 1) \times 10^{-3}$ & $0.24 \pm 0.01$ \\
\hline
\end{tabular}

${ }^{a}$ The value of $b$ is fixed, $b=0.5$, to fulfill the power law for the time dependence, respectively, of the length coordinate $z$ and the velocity $v$ of the MIMIC data. ${ }^{b}$ The error values are evaluated with a $95 \%$ confidence level established by the nonlinear regression fitting routine.

the high-temperature limit $(T \rightarrow \infty)$; and $D$ is related to the fragility, thus being connected either with the cooperative dynamics in the liquid (kinetic fragility) or with the heat capacity in a glassy state (thermodinamic fragility). ${ }^{35,36}$

By introducing the VFT temperature dependence of viscosity (eq 5) into the dynamics eqs 2 and 4, we obtain

$$
z(t)=\left(\frac{2 \Delta p G}{\eta_{0}}\right)^{1 / 2} \exp \left(-\frac{1}{2} \frac{D T_{0}}{T-T_{0}}\right) t^{1 / 2}=A_{z}(T, F) t^{1 / 2}
$$

and

$$
v(t)=\frac{1}{2}\left(\frac{2 \Delta p G}{\eta_{0}}\right)^{1 / 2} \exp \left(-\frac{1}{2} \frac{D T_{0}}{T-T_{0}}\right) t^{-1 / 2}=A_{\mathrm{v}}(T, F) t^{-1 / 2}
$$

In the coefficients $A_{i}(T, F)$, with $i=z, v$, we include the functional dependence on the temperature $T$ and on the substrate treatments $F$, which can affect the capillary pressure through the variation of the interfacial free energy and, in case, of the geometry. In this paper, we just consider the flow dynamics on untreated surfaces.

\section{RESULTS AND DISCUSSION}

Equations 6 and 7 well describe the fluid dynamics of the PU inside a microchannel filled by capillary effects. As shown in Figure 2a and Figure 2b, a regression fitting procedure on the MIMIC data set, at different temperatures, fulfill the 0.5 power model with the coefficients $A_{z}$ and $A_{\mathrm{v}}$ of eqs 6 and 7 reported in Table 1.

Clearly, the dependence on a large number of poorly known parameters in the equations of the fluid motion, eqs 6 and 7, does not make the evaluation of the structural parameters quantitatively exact.

Moreover, a direct investigation of the glass-forming characteristic parameters needs to consider the nonlinear mechanical properties for a viscoelastic fluid, with the introduction of tensorial material functions. These drawbacks can be eliminated by considering experimental data sets at different temperatures and writing a set of ratio functions, respectively, at two different temperatures. This procedure allows us to simplify the analytical expressions and to highlight the functional dependence on the structural parameters $D$ and $T_{0}$.

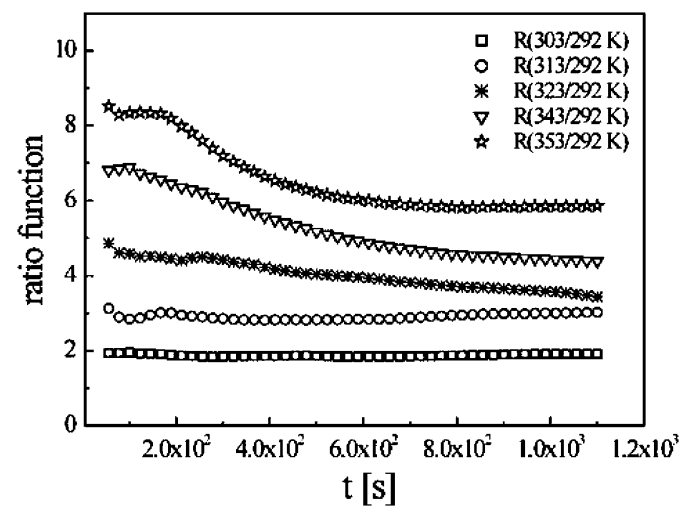

Figure 4. Ratio functions $R\left(T_{i} / T_{r}\right)$, where $T_{r}=292 \mathrm{~K}$, obtained by the experimental MIMIC data. We have considered the functions $R_{i r}$, $\forall i \neq r$, normalized with respect to the room temperature.

The most general expression of a ratio function $R_{i j}$ at two different temperatures is

$$
\begin{aligned}
& R\left(T_{i} / T_{j}\right)= \frac{z_{T i}(t)}{z_{T j}(t)}=\exp \left(-\frac{1}{2} \frac{D T_{0}}{T_{i}-T_{0}}+\frac{1}{2} \frac{D T_{0}}{T_{j}-T_{0}}\right)= \\
& \exp \left(-\frac{1}{2} D T_{0} \frac{T_{j}-T_{i}}{\left(T_{i}-T_{0}\right)\left(T_{j}-T_{0}\right)}\right)= \\
& \exp \left(-\frac{1}{2} D T_{0} \frac{\left(T_{j}-T_{i}\right)}{\left(T_{0}^{2}-\left(T_{i}+T_{j}\right)-T_{i} T_{j}\right)}\right)=\frac{A_{z i}}{A_{z j}}
\end{aligned}
$$

where $A_{z i}$ and $A_{z j}$ are the fit parameters at the temperatures $T_{i}$ and $T_{j .}{ }^{46}$

All these ratio functions, in Figure 4, are calculated with respect to the room temperature, $T_{r}=292 \mathrm{~K}$, curve, thus obtaining systems of all the possible combinations of equation pairs.

$$
\left\{\begin{array}{l}
D T_{0}=\frac{2}{\left|T_{r}-T_{i}\right|} \ln R\left(T_{i} / T_{r}\right)\left[T_{0}^{2}-\left(T_{i}+T_{r}\right) T_{0}+T_{i} T_{r}\right] \\
D T_{0}=\frac{2}{\left|T_{r}-T_{j}\right|} \ln R\left(T_{j} / T_{r}\right)\left[T_{0}^{2}-\left(T_{\mathrm{j}}+T_{r}\right) T_{0}+T_{j} T_{r}\right]
\end{array}\right\}
$$

where $R\left(T_{i} / T_{\mathrm{r}}\right)=A_{z i} / A_{z r}$ as in eq 6 . By solving these systems, we obtain for each of the eq 9 a set of values $\left(T_{0}, D\right)$, in such a way that the average values of $T_{0}$ and $D$ turn out to be $T_{0}=242.6$ $\mathrm{K}$ and $D=1.9$, respectively.

The results in Table 2 underline a difference by $15 \%$ with the values determined by fitting, with the VFT viscosity model eq 5 , the experimental rheological data of $\eta(T)$ at different temperatures and at the same shear rate of the MIMIC process, defined as $\dot{\gamma} \simeq$ $v / r$ (see Figure 5). We believe that the differences in the evaluated parameters, by MIMIC and viscometry, cannot be easily explained, because of no trivial dependence of a capillary process on numerous factors. What can be safely highlighted is the following: (i) The structural parameters of a polymeric solution strongly depend on the typical internal pressure of the device. In particular,

(46) In our current demonstration, we have just worked out the calculations with $z(t)$. However, the extension to the fluid velocity $v(t)$ could be accomplished in a straightforward manner. 
Table 2. Structural Rheological Parameters of PU Carried Out by Two Different Techniques ${ }^{a}$

\begin{tabular}{lll} 
technique & \multicolumn{1}{c}{$D$} & \multicolumn{1}{c}{$T_{0}(\mathrm{~K})$} \\
MIMIC & 1.9 & 242.6 \\
viscometry $^{b}$ & $2.3 \pm 0.2$ & $213 \pm 2.4$
\end{tabular}

${ }^{a}$ The first one, indicated as MIMIC, refers to the direct analysis of the driving flow of the PU fluid inside a capillary network. With viscometry, it is rather considered the results of the cone-and-plate rheometric analysis. ${ }^{b}$ In this case, the structural parameters has been obtained by using eq 5 with the non-Newtonian viscosity data, carried on at different temperatures $T(\mathrm{~K})$ and at fixed shear rate $\dot{\gamma}\left(\mathrm{s}^{-1}\right)$.

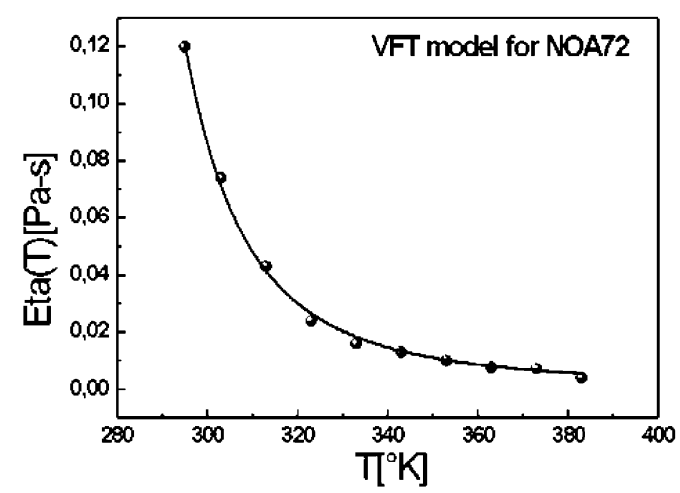

Figure 5. Experimental data $(\bullet)$ of the viscosity versus temperature, $\eta(T)$, carried on PU with the cone-and-plate rheometer at a fixed shear rate $\dot{\gamma}\left(\mathrm{s}^{-1}\right)$ value. The continuous fitting line is obtained with the VFT model, in eq 5 , for the $T$-dependence of the viscosity.

by increasing the pressure the value of the Vogel temperature $T_{0}$, in the VFT model, increases while the fragility $D$ decreases. ${ }^{36}$ This is in agreement with our results. According to eq 1, the internal pressure in the microchannel, in fact, increases as the channel size decreases. (ii) The different fabrication processes affect both the roughness and chemical surface properties, thus changing the wettability and in turn the capillary pressure according to eq 1. (iii) Finally, we should consider that a spontaneous imbibition flow in a microfluidic setup is also characterized both by a resistance to a stretching rate due to a deformation of the polymer coils and consequently by a timedependent extensional viscosity $\eta_{\mathrm{e}}$. Conversely, in the cone-andplate environment, the measured parameter is essentially a shearing viscosity $\eta_{\mathrm{s}}$.

All these factors underline the relevance of measuring the viscoelastic properties at the real working conditions.

\section{CONCLUSION}

We have succeeded in developing a microfluidic approach to assess the structural rheological parameters of a glass-forming liquid under real operation conditions, during its driving process in a micrometer capillary network, and to simplify the theoretical analysis.

Two main aspects of a direct microfluidic investigation of a polymer solution have to be addressed. First, the need to provide an exact in situ control of the dynamics of the different streams flowing in a capillary is fundamental to the fabrication and design of a large class of micro- and nanofluidics devices, such as microscopic control and memory elements, ${ }^{4}$ based on nonlinear inertial flow phenomena. In fact, when the channels are reduced in the sub-100- $\mu \mathrm{m}$ range, the flow pattern, for example, can change significantly both with the roughness or chemistry of the surface and with the flow rate. In this connection, the aim is manifold: the internal pressure has to be monitored because of its strong effects on the polymer structural parameters; the fluid direction can be controlled both by the surface treatments and by the temperature gradient. ${ }^{36,38,39}$

Besides, the need to characterize the singular behavior of a viscoelastic fluid, with nonlinear mechanical properties, directly in the final device is fundamental. For a polymeric solution, in confined environments, the elastic forces can determine an irregular dynamics even at low velocity and low Reynolds numbers. This involves elastic turbulence and a reduction of the filling velocity in the flow due to the Weissenberg effects. ${ }^{24,28}$

Finally, it has been shown that the stretching of the polymer coils in a capillary flow, have their macroscopic manifestation in a normal stress, superimposed on the Laplace pressure and opposed to the fluid motion.

All in all, the method described in this paper can be considered as a valid tool for the direct investigation of the structural properties of particular nonlinear viscoelastic fluids in confined microscopic systems.

\section{ACKNOWLEDGMENT}

The authors gratefully acknowledge Dr. F. Lionetto for helpful discussions and for valuable experimental support with the rheometric measurements and S. D'Amone for valuable technical assistance.

Received for review April 26, 2004. Accepted October 18, 2004.

AC049381F 